\title{
Highly Birefringent Nonlinear PCF for Optical Sensing of Analytes in Aqueous Solutions
}

\author{
Huseyin Ademgil ${ }^{1}$ and Shyqyri Haxha ${ }^{2}$
}

1 Department of Computer Engineering, European University of Lefke, Gemikonagi-Lefke, KKTC, Mersin 10, Turkey E-Mail: hademgil@eul.edu.tr

2 Department of Computer Science and Technologies, University of Bedfordshire, University Square, Luton, Bedfordshire, LU1 3JU, United Kingdom, E-Mail: Shyqyri.Haxha@ beds.ac.uk

\begin{abstract}
This paper presents a design of a nonlinear Photonic Crystal Fiber (PCF) based sensor exhibiting simultaneously high sensitivity, high birefringence and low confinement losses for liquid analyte sensing applications. We investigate the PCF sensor performance for the following analytes; Water, Ethanol and Benzyne. The impact of various design parameters of the highly nonlinear PCF on the relative sensitivity, the confinement losses and the birefringence features of the proposed PCF structure is numerically investigated by employing the full vectorial Finite Element Method (FEM). According to our FEM numerical results, a three ring nonlinear PCF based sensor is designed that simultaneously offers high birefringence of order $10^{-3}$ and high relative sensitivity at wide wavelength range.
\end{abstract}

Keywords -Evanescent Photonic Crystal Fiber, Integrated Optics, Optical Sensor,

\section{INTRODUCTION}

In recent years, Photonic Crystal Fibers (PCFs) are particularly used for sensing applications due to their unique abilities that cannot be obtainable by conventional optical fibers. The advancement of PCF design technology as well as their far-reaching abilities renders them a potentially excellent solution for nanotechnology mechanisms for use in many optical applications [1-8].

The elements of light propagation and inherent geometric flexibility of PCFs enable researchers to realize non-traditional propagation features. This is achieved by generating and adapting the size, architecture and positioning of the core and cladding holes. A wealth of research has been dedicated to such microstructured fibres and has concluded that atypical dispersion behavior [1], endlessly single-mode guidance [7], light guidance in low-index components [9, 10], high levels of birefringence [11], and modified non-linear properties can all be attained across broad portions of the wavelength spectrum. Such individualistic features within PCFs have facilitated subsequent enhancements in the domain of optical communications [1], non-linear optics [12], high power technologies [4], as well as sensing [2, 3, 13].

Sensors based on optical fibres act as inexpensive and efficacious resolution as they are exceptionally sensitive, small, robust, and bestow the ability to monitor remotely [14]. In PCFs, hollow-core Photonic Band Gap (PBG) structures may be ideal for a selection of sensingapplication solutions [13]. But the complicated production procedure for PBG PCFs has resulted in a downfall in the importance of sensing devices. On the other hand, PCFs featuring index guidance operations are optimal for applications on sensing [2]. 
Sensing utilization in index-guided PCF sensors are based on evanescent interaction occurring between guided electromagnetic light mode and a sample (Liquid / Gas), resembling that of traditional optical fibre sensors $[2,14]$. This evanescence in domains where light propagates in small spaces emanates to portions in which the sensing analyte under observation is located. The existence of air holes within the cladding allows for biological/chemical samples to be in the immediate vicinity of the PCF core, either in a liquid or gaseous state $[2,9,13]$. In recent years, the concept of confined light guidance through analyte (gas and/or liquid) would provide a key step forward to explore the essential nature of interactions between the analyte and the light propagation. Given that PCF sensors allow for much interaction throughout the fibre and a given chemical sample located in the air holes, they are highly useful in identifying bacteria and remote sensing $[6,10,15,16]$. Generally, alcohol or water are the primary liquids utilized in these processes given that they provide large volumes of bio/chemical solutions.

In PCFs, as discussed prior, the typical process for identifying the low index material is depends on the interaction of the evanescent field of the guidance conditions with the relevant gas or liquid being analysed/detected $[2,9,16]$. Electromagnetic forces acting upon this analyte under scrutiny can be strengthened as necessary. To date, many research papers have aimed to provide a lucid understanding of the sensitivity aspects in applying PCFs in a multitude of liquid or gas analytes $[2,9]$. Evanescent field sensing whereby both the core and cladding are microstructured was first introduced by Ref.[13] and analysed in detail by Cordeiro and colleagues [2]. Authors [2] studied a range of low index analytes for PCF sensitivities. Recently, enhancements of relative sensitivities resulting in minimised loss have been made through use of an octagonal PCF structure [16]. Also, Yu et. al. [17] constructed a hyper-sensitive PCF temperature sensor in which the holes of the core contain ethanol. Researchers in [17] showed their sensor to be easy in application and readily capable, in addition to being inexpensive to synthesise. Liu and colleagues [10] suggested PCF structures for use in sensing water-levels whereby the PCF is constructed to be highly perceptive to temperatures of the surrounding environment.

In some particular fiber optic sensors, maintaining polarisation states can be crucial. Traditional Single Mode Fibres (SMFs) that feature circularly-symmetric cores are unable to sustain electromagnetic polarisation configurations for many reasons, as delineated by [18]. The polarisation conditions may be sustained through application of modal birefringence. A widely used approach for this method in PCFs is adjusting the air hole diameter or through contorting the shape of the air holes (elliptical air holes) which surrounds the core [11]. Some of the most contemporary research $[11,19]$ has allowed us to understand and execute PCF designs inclusive of large birefringence to the order of $10^{-3}-10^{-2}$.

It is imperative to make the distinction that, different to typical approaches to maintaining polarisation fibres, namely bow-tie, elliptical core or Panda which comprise of at least two glasses with individualized thermal expansion coefficients, birefringence is attainable in PCFs that are largely insensitive to temperatures. This is a fundamental feature of many processes. Adjustable and refined high birefringence PCFs may be garnered through symmetrical distributions of the relevant sensory material (Liquid/Gas) into the holes. Given the introduction of a new functional element, magnetic substances have been noteworthy in their infiltration of PCF as the refractive index is highly perceptive to the external magnetic field [20]. 
In addition, PCFs that bestow exceptional levels of sensitivity and birefringence have been analysed for various sensing uses [21, 22]. Strain and temperature sensitive PCFs were investigated in [21], and constructed via Ge-doped silica rods placed on the PCF's $x$-axis. Conversely, birefringence as applied in PCFs to sustain polarisation parameters as a function of temperature have been analysed in practice in Ref. [22].

Observation of structures by Yang et. al. [5] have revealed a nonlinear PCF structure with high birefringence and low confinement. In this paper [5], simulations revealed high birefringence of around $10^{-2}$, high non-linearity and minimal confinement losses can all be achieved in the demonstrative structure. Authors in Ref. [5] have suggested that such proposed PCF structure can be useful for sensing applications.

In this paper, evanescent, hexagonal PCF structures featuring additional air holes about the PCF core are put forward and analysed for their sensing abilities. To attain the appropriate levels of birefringence, sensitivity (maximal) and confinement losses (minimal) concurrently, the design used in this study features circular air holes both in the cladding and core domains. Again, as delineated by Ref. [5], a proposal for PCF constructions that feature additional air holes in the aforementioned areas enhances birefringence and non- linearity. The sensitivity of the current model requires further investigation and improvement in order to purport it as a viable model. Therefore, in this study, we draw on foundations provided by Cordeiro et. al. [2] have been utilised in expressing a model and Yang et. al. [5] to implement it. To better understand the implications of air holes, a detailed study has been conducted. Sensitivity, birefringence and the confinement losses of the proposed non-linear PCF structure are studied in detail through application of differing liquid analytes.

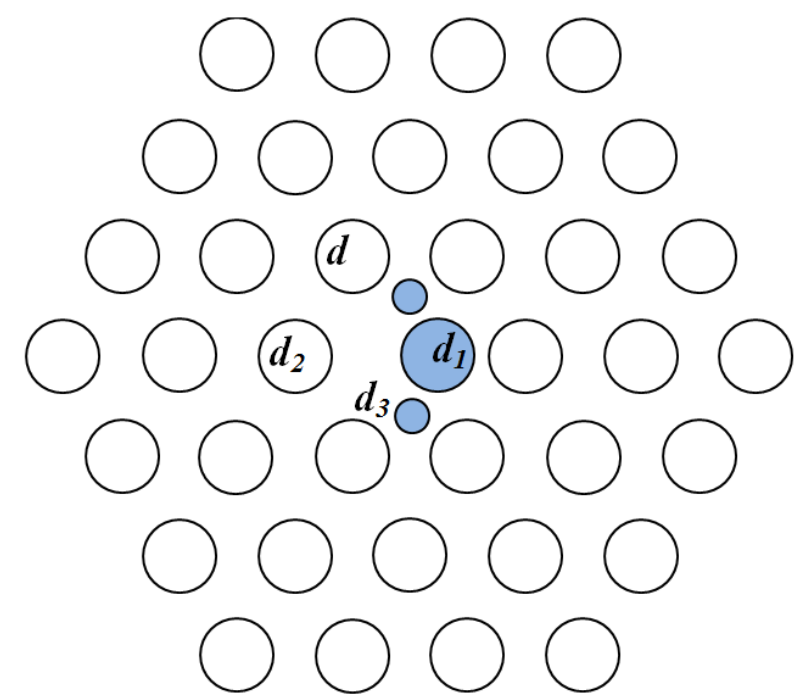

Fig. 1 Schematic diagram of the proposed PCF with supplementary liquid filled air holes in the core region.

Fig.1 demonstrates a representation of the preliminary model, in which three additional air holes in the core domain and 36 of larger size in the cladding domain are organised in triangular formation across the silica backdrop. It is evident that the three variables of non-linearity, 
birefringence and confinement loss in a PCF is proportional to the foci and asymmetries of the fibre configurations, which are dependent on the size and location of holes in the cladding domain [5]. Additionally birefringence can be manipulated via holes located in the initial ring. Conversely, PCF perceptiveness is relative to confinement losses and direct interactions of light, as well as the sample within the core domain [2].

In this study, the numerical analysis of the proposed PCF structure has been carried out using a commercial Full Vectorial Finite Element Method (FVFEM). The FVFEM is an all-purpose, efficient numerical technique to obtain approximate solutions to boundary value problems [1-2, 7 , 16]. Moreover, FVFEM is an accurate, reliable and well tested method for modal analysis. This numerical method is highly accurate and efficient to design PCFs where by defining the subdomains and boundary conditions with correct parameters and we can solve complex design problems for both Electric field and Magnetic field [23]. The accuracy of the FVFEM method can be improved by increasing the mesh density [23]. In the following section, a thorough design process and modelling analyses of PCFs is undertaken using the stated FVFEM.

\section{DESIGN AND MODELLING OF THE PCF STRUCTURE}

Several problems are faced in designing, optimizing and generating photonic waveguides, the central regions of which are made of liquids/gases. These issues essentially pertain to discovering appropriate cladding materials which have a very small refractive index so as to support the waveguiding. In practical scenarios, negative dispersion may be exhibited by optical nonlinearity. In addition, there is a critical discrepancy between optical waveguides and traditional silica optical fibres and this leads to considerable limitations in practical uses. Hence, when liquid insertion at central hole is permitted, it is possible that defect-less silica PCFs may offer a practical alternative solution for low-refractive-index liquid cores [24]. There can be comprehensive regulation of the PCFs effective cladding index when fine-tuning of the hole diameter (d) and hole to hole spacing $(\Lambda)$ of the hole arrays in the cladding $[6,25]$. Furthermore, different techniques for selectively filling the air gaps in hexagonal PCFs with liquids have been developed recently and are now being used commonly in laboratory tests $[6,26]$. Filling the central hole of a PCF structure with analyte like liquid allows the incident light to pass directly though the liquid due to the effective index guidance [6].

This study takes into account the conveniences of index-guiding mechanism and the selective filling method to find out the design factors of the suggested PCF. The authors have suggested a structure in which the hole to hole space is kept at $\Lambda=2 \mu \mathrm{m}$ and the supplementary air holes are sized at $0.36 \Lambda$ and $0.465 \Lambda$ [5]. The experimental perspective suggests that the risk of collapse between air holes while carrying out the fabrication process, along with the single mode operation, can be reduced by using larger hole to hole spacing. Hence, we suggest that the hole to hole spacing should be kept at $\Lambda=2.4 \mu \mathrm{m}$ in the suggested model, and the supplementary air hole diameters filled with analytes should be somewhat larger than the PCF structure given in [5]. According to our research, these parameters will greatly reduce the complexity of the probable fabrication procedure.

The suggested PCF structure along with the supplementary air holes in the central region can be seen in Figure 1. Within the cladding, the air holes have a diameter of $d=1.5 \mu \mathrm{m}$. In the core region, the three air holes that have been filled with analyte are represented by $d_{1}$ and $d_{3}$. Higher 
birefringence levels can be attained and light analyte interaction improves when the air hole in the foremost ring of the cladding is used. Here, the diameter is represented by $\mathrm{d}_{2}$. The sizes of the upper and lower supplementary air holes $\mathrm{d}_{3}$ are similar and are situated on the $y$-axis and have a fixed distance of $d_{3} 1.2 \mu \mathrm{m}$. In contrast, the bigger supplementary core air hole $\left(\mathrm{d}_{1}\right)$ is moved horizontally over the x-axis by a fixed distance $0.6 \mu \mathrm{m}$ away from the centre of the PCF. 36 air holes are present in the suggested PCF structures' cladding region and the refraction index of the cladding holes is fixed at 1 . Silica is utilized as the background material and has a refraction index of 1.45 . There will be powerful light confinement when there is variation in refractive index of the core and the cladding.

This study utilizes the FV-FEM to examine the suggested PCF structures. The basis of the FVFEM development for the modal assessment is the inclusion of the anisotropic Perfectly Matched Layers (PMLs) which has the ability to manage several modes as needed and evaluate the leaky modes $[1,7,16]$. The PML is strictly speaking not a boundary condition but an additional domain that absorbs the incident radiation without producing reflections. The modal assessments are carried out on the cross-section in the $x$ - $y$ plane of the PCF as the wave moves along in the $z$-direction.

The confinement loss $L_{c}$, in decibels per meter is given by;

$$
L_{c}=\frac{40 \pi}{\ln (10) \lambda} \operatorname{Im}\left\{n_{\text {eff }}\right\}
$$

where, $I_{m}\left\{n_{e f f}\right\}$ is the imaginary part of the effective refractive index of the mode [1].

The birefringence is determined by taking the real part of the effective index difference of the two fundamental polarisation modes $H E_{11}^{x}$ and $H E_{11}^{y}$ and can be written as [11];

$$
B=\left|n_{e f f}^{x}-n_{e f f}^{y}\right|
$$

In PCF structures, the relative sensitivity coefficient $r$ can be calculated by [2]:

$$
r=\frac{n_{r}}{n_{e}} \times f
$$

where, $n_{r}$ is the refractive index of the absorbing material and $n_{e}$ is the real part of effective refractive index, $f$ is the percentage of energy presents in the holes. According to the Poynting's theorem $f$ can be expressed as [2]:

$$
f=\frac{(\text { sample }) \int \operatorname{Re}\left(E_{x} H_{y}^{*}-E_{y} H_{x}^{*}\right) d x d y}{(\text { total }) \int \operatorname{Re}\left(E_{x} H_{y}^{*}-E_{y} H_{x}^{*}\right) d x d y} \times 100
$$

\section{SIMULATION, RESULTS AND DISCUSSIONS}

In this study we have investigated the PCF structure in terms of sensitivity where supplementary core holes are filled with various analytes. Three different analytes are investigated whose refractive index values are obtained as Water $(\mathrm{n}=1.33)$, Ethanol $(\mathrm{n}=1.354)$ and Benzyne $(\mathrm{n}=1.366)$ [27]. It is essential to state that the proposed PCF structure supports only four non- 
degenerate eigenmodes $\left(H E_{11}^{x}, H E_{11}^{y}, H E_{21}^{x}\right.$ and $\left.H E_{21}^{y}\right)$ over a very wide wavelength range. In PCFs, due to non-circular core structure these modes are linearly polarized and categorized as $L P$ modes. In this respect the fundamental $L P_{01}$ mode corresponds to $H E_{11}$. It is worth to note that only the fundamental $L P_{01}$ modes are considered.

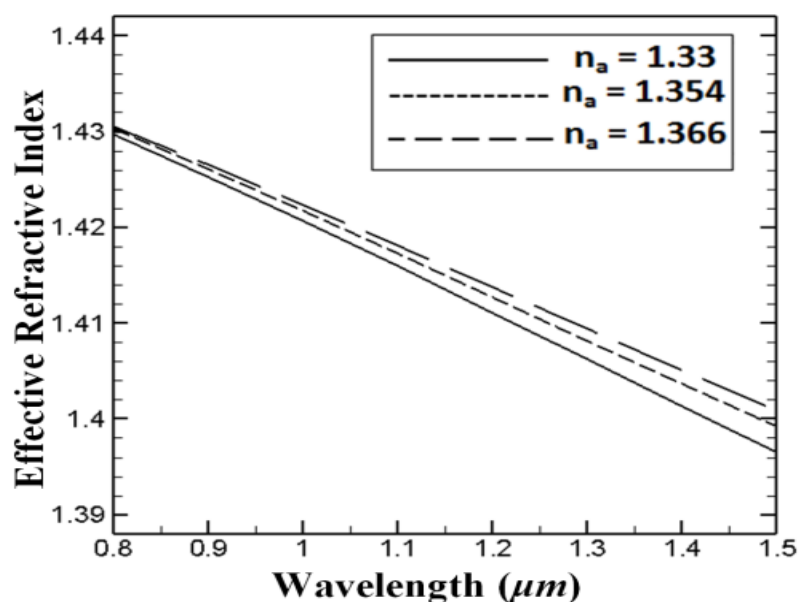

(a)

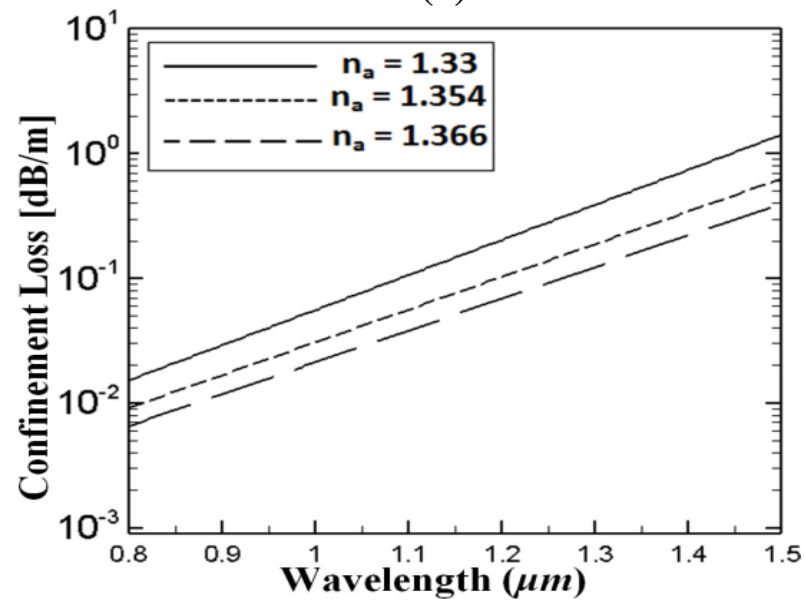

(c)

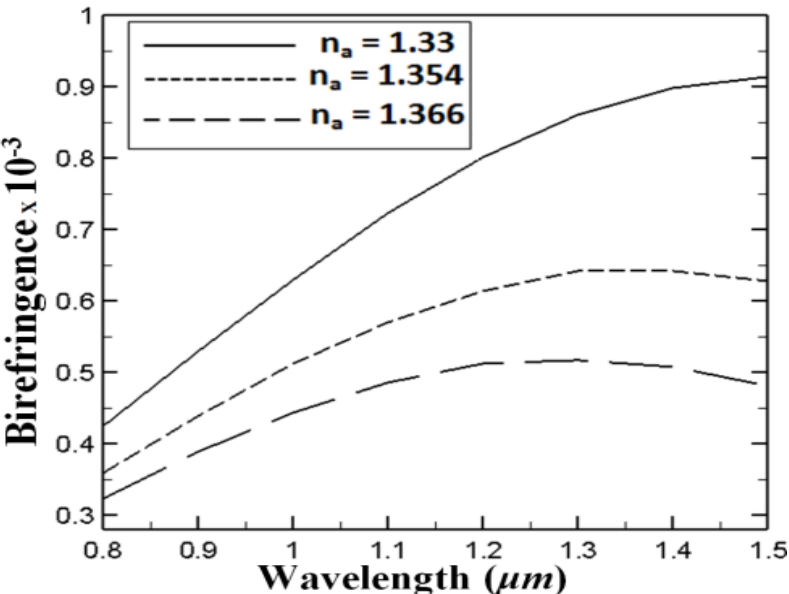

(b)

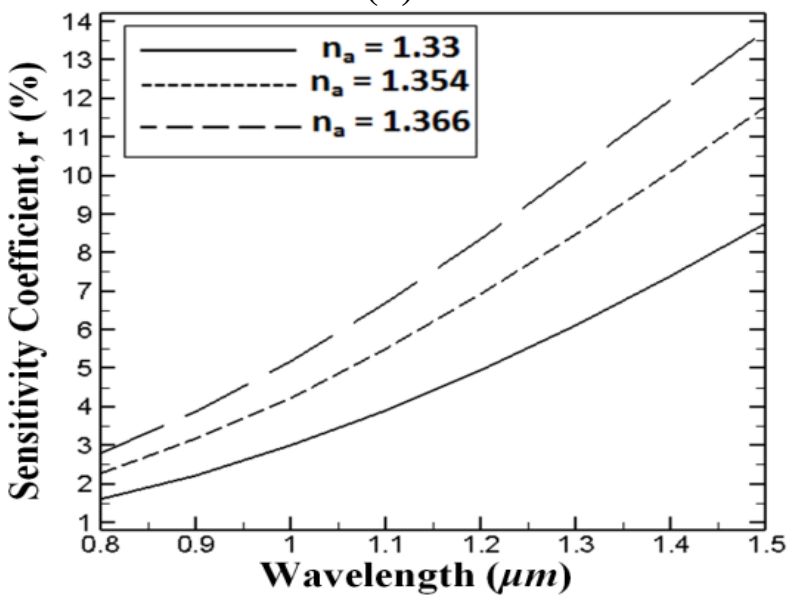

(d)

Fig. 2 Variation of the Effective refractive index (a), the modal birefringence (b), confinement loss (c) and the sensitivity coefficient (d) as a function of the operating wavelength, where $d=$ $\mathrm{d}_{1}=\mathrm{d}_{2}=1.5 \mu \mathrm{m}$ and $\mathrm{d}_{3}=0.5 \mathrm{~d}$.

Initially, the impact of the operating wavelength on the effective refractive index, birefringence, and confinement loss and sensitivity coefficient of the proposed PCF structure is illustrated in Fig. 2 (a)-(d). The PCF design parameters are set as follows; $\Lambda=2.4 \mu \mathrm{m}, \mathrm{d}=\mathrm{d}_{1}=\mathrm{d}_{2}=1.5 \mu \mathrm{m}$ and $\mathrm{d}_{3}=$ $0.5 \mathrm{~d}$. It can be seen from Fig. 2 (a) that the effective refractive index of the fundamental $L P_{01}$ $\left(H E_{11}^{x}\right)$ mode is decreasing with increasing the wavelength for all types of analytes used in this study. On the other hand, in Fig. 2 (b), it can be seen that the lower index analyte (water) is reaching much higher birefringence when compared to ethanol and benzyne. A similar trend behavior of the analytes under this study can be realised when the confinement losses are examined as a function of the wavelength. In other words, our simulations show that higher index analyte, in the 
core region, reduces the confinement losses in wide wavelength range. The above findings were investigated by Ref [5]. However, the sensitivity of the proposed PCF structure for various liquid analytes still needs to be computed and optimized before one can conclude that it can be deployed in practical applications.

Our simulation results show that proposed PCF exhibits greater sensitivity to the higher index analytes. This can be related to the fact that the fundamental mode is more confined in the core area for the higher index analytes. As fundamental mode is more confined in the core region, the electromagnetic power interaction between the light and the analyte increases, as a result of this, we obtain a higher sensitivity coefficient. As can be seen from Fig. 2(c) and (d), the relative sensitivity coefficient of the proposed PCF structure is increasing where there is a significant improved mode confinement. It is evident from Fig. 2(d) that the relative sensitivity increases considerably, for all analytes under this study, as the operating wavelength increases. This increase can be linked to the noteworthy interaction between the liquid filled core holes and the light mode at the longer operating wavelengths.

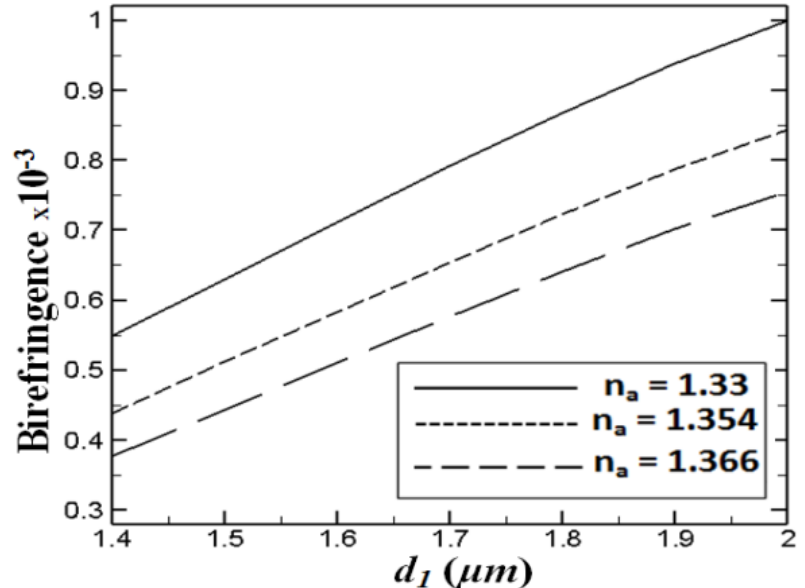

(a)

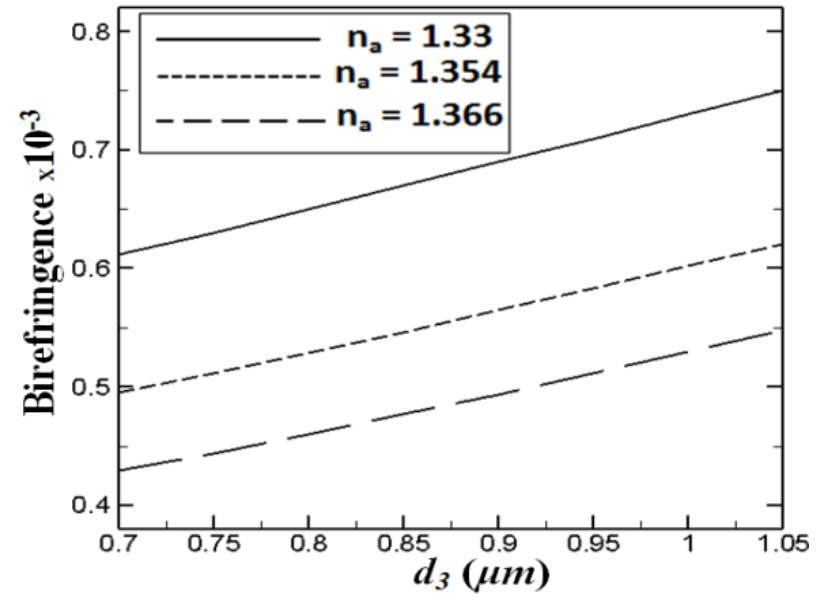

(c)

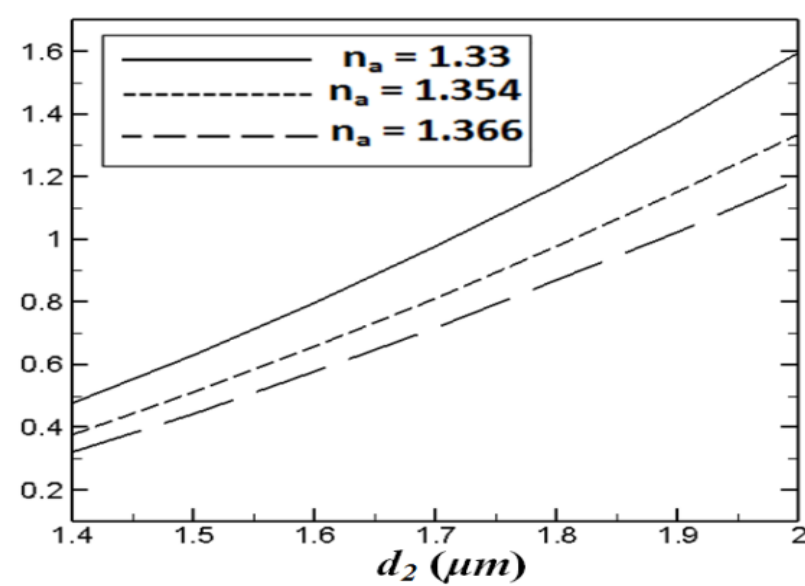

(b)

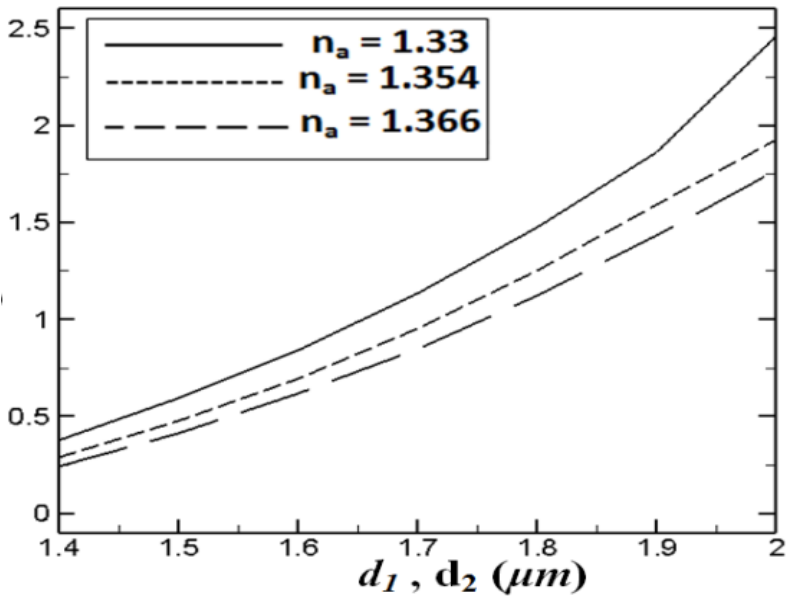

(d)

Fig. 3. Variation of the modal birefringence as a function of the (a) $d_{1}$ where, $d=d_{2}=1.5 \mu \mathrm{m}$ and $\mathrm{d}_{3}=0.5 \mathrm{~d}$ (b) $\boldsymbol{d}_{2}$ where, $\mathrm{d}=\mathrm{d}_{1}=1.5 \mu \mathrm{m}$ and $\mathrm{d}_{3}=0.5 \mathrm{~d}$, (c) $\boldsymbol{d}_{3}$ where, $\mathrm{d}=\mathrm{d}_{1}=\mathrm{d}_{2}=1.5 \mu \mathrm{m}$ and (d) $\boldsymbol{d}_{\boldsymbol{1}}$ $\& \boldsymbol{d}_{2}$, where $\mathrm{d}=1.5 \mu \mathrm{m}$ and $\mathrm{d}_{3}=0.5 \mathrm{~d}$ at $\lambda=1 \mu \mathrm{m}$. 
Previously, Yang et al. [5] have presented the impact of the cladding air hole diameter, $\mathrm{d}$ on the birefringence and confinement losses. It has been found that the birefringence and confinement losses are decreasing considerably with the increase in the air hole diameter. Our FEM simulations have confirmed the findings reported by the authors in Ref. [5]. Meanwhile, virtuous light confinement causes higher light analyte interaction. Therefore, the sensitivity of the proposed PCF is increasing sharply. Furthermore, our simulation results have shown that the single mode operation is directly linked to the construction geometry and the size of the air holes in the cladding region of the proposed PCF. Therefore, in our study in order to achieve single mode operation together with significant amounts of birefringence, sensitivity and low losses the optimum cladding air hole size, $\mathrm{d}$ is fixed to $1.5 \mu \mathrm{m}$.

In order to observe the impact of the core holes on the propagation characteristics of the proposed PCF sensor, we have investigated the key design parameter such as; $d_{1}, d_{2}$, and $d_{3}$ when the supplementary core holes $\left(\mathrm{d}_{1}\right.$ and $\left.\mathrm{d}_{3}\right)$ are filled with various analytes.

The influence of parameters $d_{1}, d_{2}$, and $d_{3}$ on the PCF birefringence is illustrated in Fig. 3 (a), (b) and (c). As can be seen from our simulation results presented in these figures, the birefringence is increasing when the refractive index of the analytes, under study, is decreasing. By comparing results shown in Fig. 3 (a), Fig. (b) and Fig.3 (c), one can evidently see that the size of the $d_{2}$ has superior impact on the birefringence, whereas the influence of the $d_{3}$ is insignificant. In order to further optimise the PCF obtained results, we have increased the diameter of $\mathrm{d}_{1}$ and $\mathrm{d}_{2}$ concurrently, and results are illustrated in Fig. 3 (d). As it can be observed from this figure, the birefringence magnitude for all three analytes under study has increased almost $15 \%$ compared to results obtained in Fig. 3 (b).

The field confinement and its deterioration rate have a central role in the propagation properties of the proposed PCF structure. The confinement loss of the proposed PCF sensor is one of the most critical parameter in the practical applications. Variation of the confinement loss as a function of diameters $d_{1}, d_{2}$, and $d_{3}$ for various analytes are illustrated in Fig. 4 (a), (b), (c) and (d). It may be seen from these figures that confinement losses are decreasing when the refractive index of the analytes are increasing. Figure 4 (a), (b), (c) shows that the dimensions of each $\mathrm{d}_{1}, \mathrm{~d}_{2}$, and $\mathrm{d}_{3}$ have similar impact on proposed PCF confinement losses. However, a sharp increase can be realised when the diameter of $\mathrm{d}_{1}$ and $\mathrm{d}_{2}$ is increased concurrently.

As it has been presented in Ref. [5], the confinement loss of the proposed PCF structure can be minimised by increasing the number of air hole rings in the cladding region (see Fig. 7 in Ref. [5]). Our simulations also have verified and confirmed that the confinement losses of the proposed PCF can be reduced significantly by increasing the number of air hole rings surrounding the core without effecting the birefringence and sensitivity excessively.

Next, sensitivity feature of the proposed PCF sensor is investigated by calculating the sensitivity coefficient $r$ using eq. (3); the obtained results have been illustrated in Fig. 5. Simulation results indicate that the relative sensitivity of the PCF structure increases when supplementary core holes are filled with higher index samples. This phenomenon is due to the fact that the optical mode is 
more confined in the core region when higher index analytes are used. This phenomenon can be realised as well when we investigate the obtained results in Fig. 4. The electromagnetic power interaction between the samples and the light is increasing when the greater index analytes are used, consequently this is triggering the increase of the sensitivity coefficient.

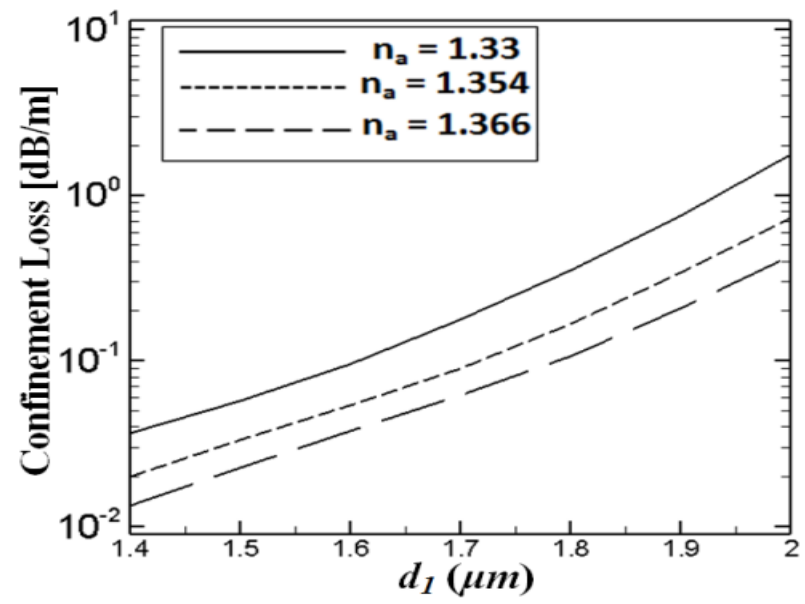

(a)

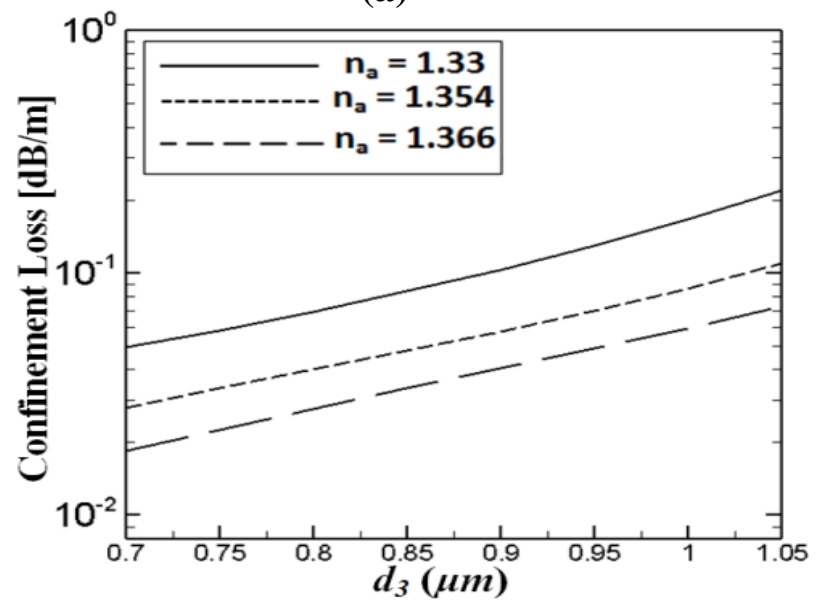

(c)

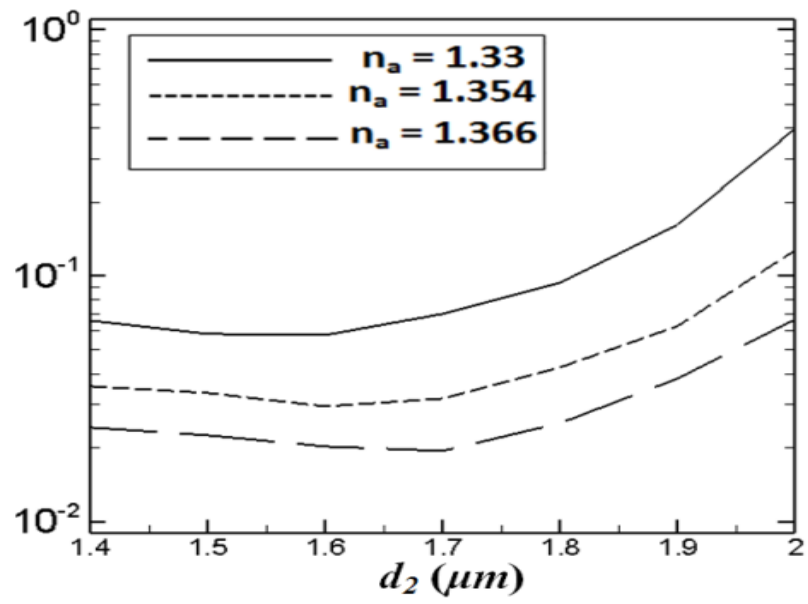

(b)

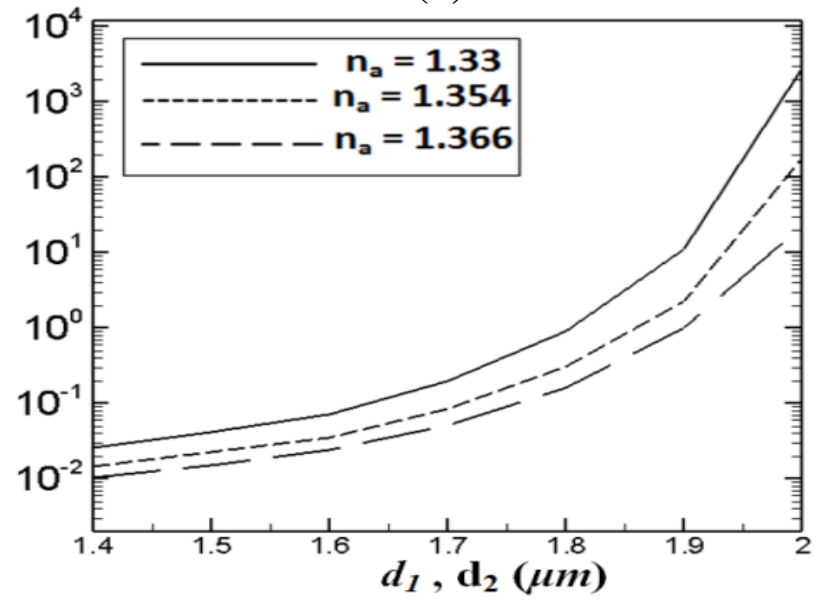

(d)

Fig. 4. Variation of the Confinement Loss as a function of the (a) $\boldsymbol{d}_{\boldsymbol{1}}$ where, $\mathrm{d}=\mathrm{d}_{2}=1.5 \mu \mathrm{m}$ and $\mathrm{d}_{3}=0.5 \mathrm{~d}$ (b) $\boldsymbol{d}_{2}$ where, $\mathrm{d}=\mathrm{d}_{1}=1.5 \mu \mathrm{m}$ and $\mathrm{d}_{3}=0.5 \mathrm{~d}$, (c) $\boldsymbol{d}_{3}$ where, $\mathrm{d}=\mathrm{d}_{1}=\mathrm{d}_{2}=1.5 \mu \mathrm{m}$ and (d) $\boldsymbol{d}_{\boldsymbol{1}}$ $\& \boldsymbol{d}_{2}$, where $\mathrm{d}=1.5 \mu \mathrm{m}$ and $\mathrm{d}_{3}=0.5 \mathrm{~d}$ at $\lambda=1 \mu \mathrm{m}$.

From Fig. 5 (a), (b), (c) and (d), it can be seen that the diameter $\mathrm{d}_{2}$ has a major influence on the sensitivity coefficient, whilst the effects of $d_{1}$ and $d_{3}$ is almost negligible. Similar trends can be observed for the birefringence, the sensitivity coefficient of the proposed PCF is increasing by roughly $12 \%$ when the diameter of $d_{1}$ and $d_{2}$ is increasing concurrently for $n_{a}=1.354$ and $n_{a}=$ 1.366. It is worth noting that the influence of the concurrent increase in the $\mathrm{d}_{1}$ and $\mathrm{d}_{2}$ are negligible when the core holes are filled with $\mathrm{n}_{\mathrm{a}}=1.33$. 
Next we investigate the field profiles of the proposed PCF structure when the sensing region is filled with analyte. Fig. 6 shows the field profiles of the dominant $E_{x}$ component of the fundamental $H E_{11}^{x}$ mode of the proposed PCF structures when the core holes are filled with ethanol $\left(\mathrm{n}_{\mathrm{a}}=1.354\right)$.

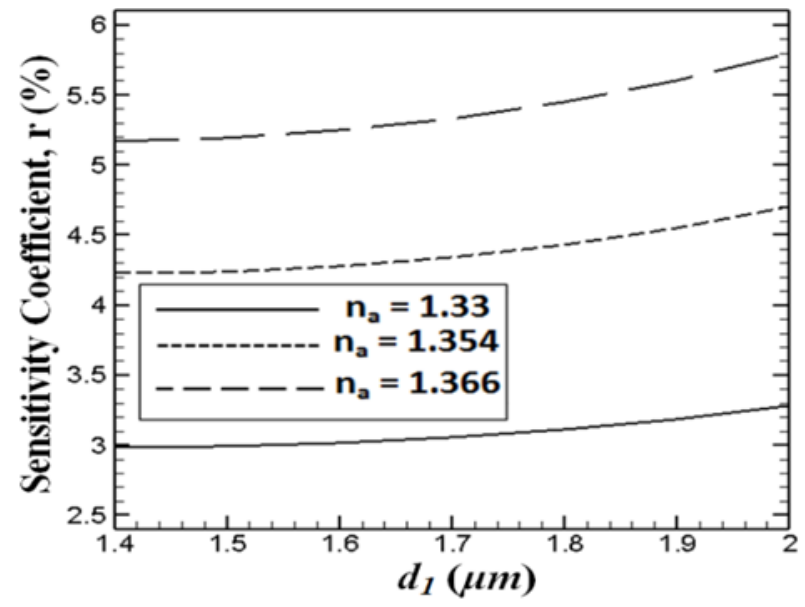

(a)

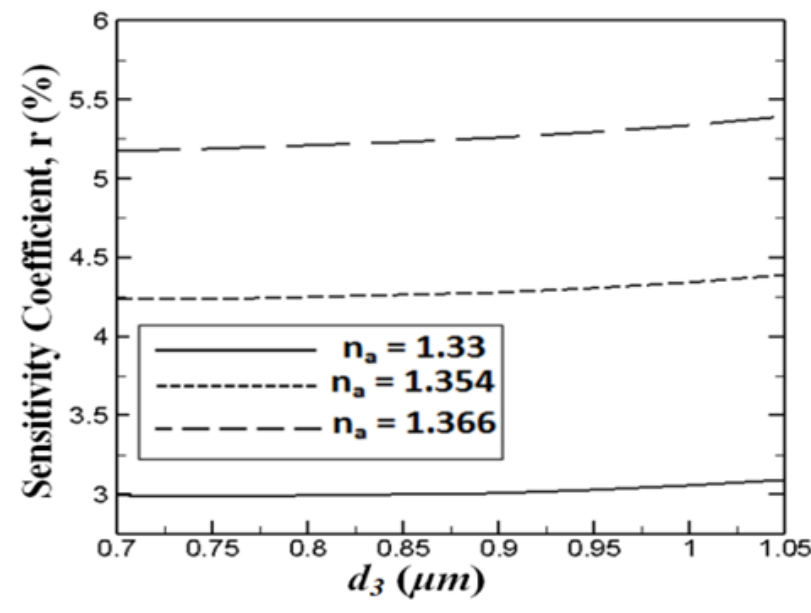

(c)

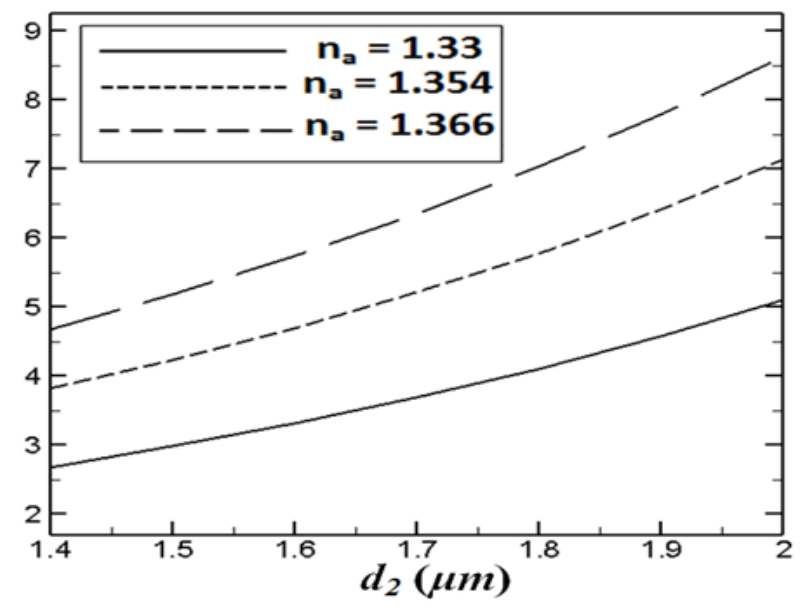

(b)

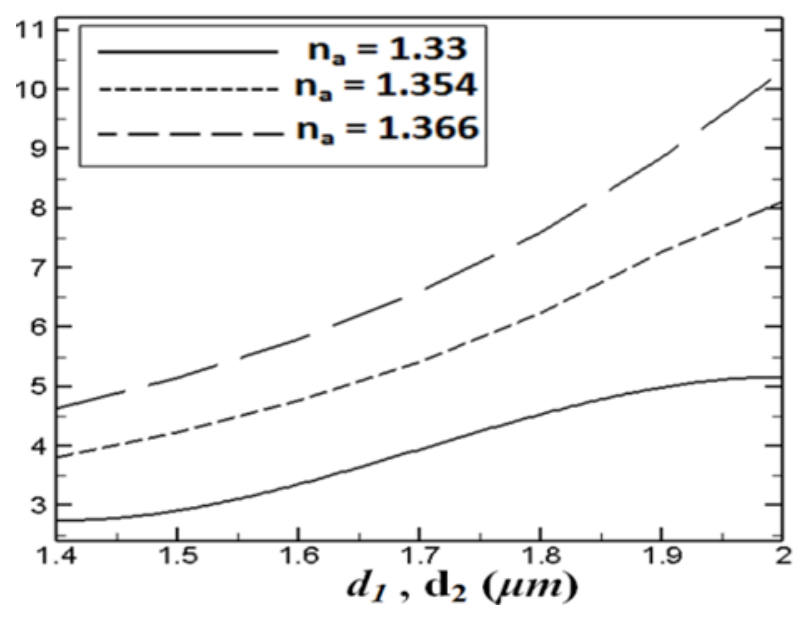

(d)

Fig. 5. Variation of the sensitivity coefficient as a function of the (a) $d_{1}$ where, $d=d_{2}=1.5 \mu \mathrm{m}$ and $\mathrm{d}_{3}=0.5 \mathrm{~d}$ (b) $d_{2}$ where, $\mathrm{d}=\mathrm{d}_{1}=1.5 \mu \mathrm{m}$ and $\mathrm{d}_{3}=0.5 \mathrm{~d}$, (c) $d_{3}$ where, $\mathrm{d}=\mathrm{d}_{1}=\mathrm{d}_{2}=1.5 \mu \mathrm{m}$ and (d) $\boldsymbol{d}_{1} \& \boldsymbol{d}_{2}$, where $\mathrm{d}=1.5 \mu \mathrm{m}$ and $\mathrm{d}_{3}=0.5 \mathrm{~d}$ at $\lambda=1 \mu \mathrm{m}$.

It can be clearly seen from Fig. 6 (a) and (b) that the electric field distribution in $x$-axes is squeezed as a result of the increase in $d_{1}$ or $d_{2}$. This will lead to an increase in birefringence and relative sensitivity. Furthermore, it can be seen from Fig. 6 (d) that the electric field is deformed and the light tends to leak towards the $y$-axes. This phenomenon is due to the increase of the birefringence, confinement losses and the sensitivity coefficient. Since, the smaller air holes, $\mathrm{d}_{3}$ are positioned at the $y$-axis, concurrent increase in the diameters of $\mathrm{d}_{1}$ and $\mathrm{d}_{2}$ are expected to increase the power interaction between the light and the analyte.

It is well known that the nonlinear coefficient is inversely proportional to the effective area [5]. Therefore, a small effective mode area leads to a high nonlinear coefficient that would be useful 
in the various applications. The nonlinear coefficient of proposed PCF structure was previously studied in Ref. [5]. It can be seen from Fig. 6 that proposed PCF structure exhibits relatively small effective mode area. Our simulations show that the nonlinear coefficients of the illustrated field profiles of $x$-polarised $H E_{11}$ mode are reported as (a) $49,4 \mathrm{~W}^{-1} \cdot \mathrm{km}^{-1}$ (b) $48 \mathrm{~W}^{-1} \cdot \mathrm{km}^{-1}$ (c) $51,3 \mathrm{~W}^{-}$ ${ }^{1} \cdot \mathrm{km}^{-1}$ (d) $50,8 \mathrm{~W}^{-1} \cdot \mathrm{km}^{-1}$. These results match well with the results presented in Ref. [5].

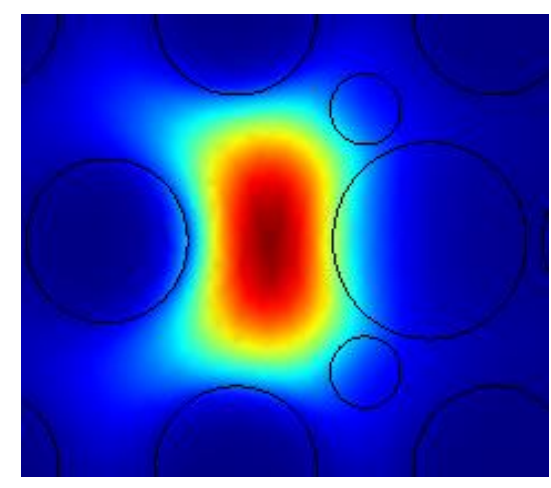

(a)

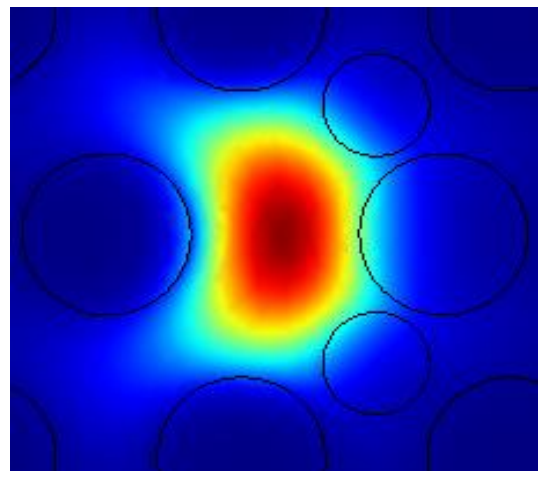

(c)

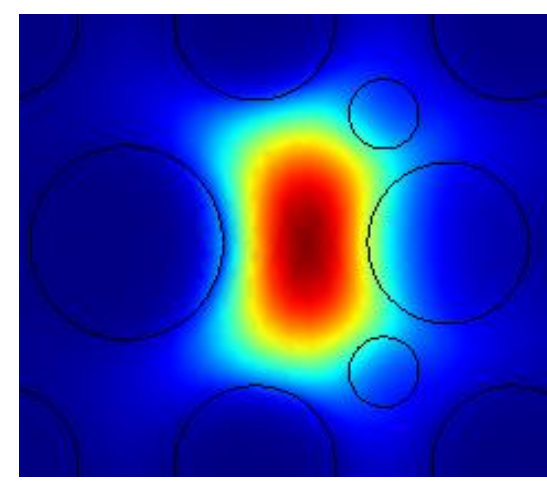

(b)

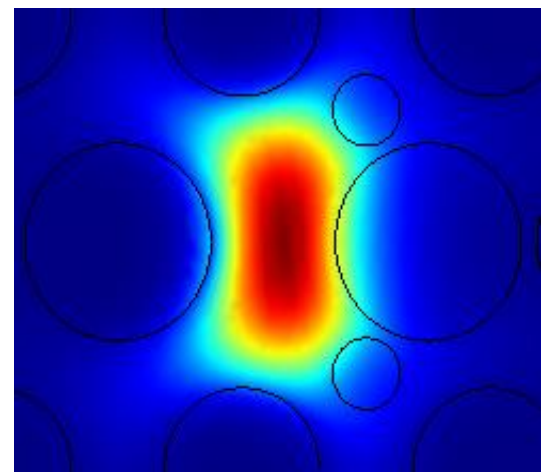

(d)

Fig. 6. The field profiles of the dominant $E_{x}$ component of the fundamental $L P_{01}$ mode with $\mathrm{n}_{\mathrm{a}}=$ 1.354 (a) $\mathrm{d}_{1}=1.8 \mu \mathrm{m}, \mathrm{d}_{2}=1.5 \mu \mathrm{m}, \mathrm{d}_{3}=0.5 \mathrm{~d}$ (b) $\mathrm{d}_{2}=1.8 \mu \mathrm{m}, \mathrm{d}_{1}=1.5 \mu \mathrm{m}, \mathrm{d}_{3}=0.5 \mathrm{~d}$ (c) $\mathrm{d}_{3}=0.95 \mu \mathrm{m}$ and $\mathrm{d}_{2}=\mathrm{d}_{1}=1.5 \mu \mathrm{m}$ (d) $\mathrm{d}_{2}=\mathrm{d}_{1}=1.8 \mu \mathrm{m}$ and $\mathrm{d}_{3}=0.5 \mathrm{~d}$. where, $\mathrm{d}=1.5 \mu \mathrm{m}$ at $\lambda=1 \mu \mathrm{m}$.

Lastly, considering the fabrication viability and its uses, it becomes imperative to evaluate and visualize the development of the suggested PCF structure. In addition, the fibre holes have to be filled with analyte/liquid such that it does not damage the operability of the fibre. The laser light should be very close to the single mode operation so as to attain the desired sensitivity. The PCF holes can be selectively filled with analytes by using a variety of methods. It needs to be mentioned that in the suggested PCF structure, the intricacy of analyte filling has shown a subtle increase, essentially in the core region. A distinct technique was put forward by Huang et al. [28] which permit selective filling of the air holes within air-core PCFs and developing different operational PCFs for various applications. The authors have particularly shown that any cladding hole or simply the supplementary core holes can be filled. In the filling process, pressure can be put on the UV-curable polymer within the PCF. Luo et al [29] and Gerosa et al. [30] have recently demonstrated experimentally that there can be efficient fabrication of the PCF structures with 
liquid filled cladding/core holes with the help of identical techniques. For this purpose, the PCF structure suggested in this paper can be fabricated with the existing nanotechnology which shows substantial benefits with respect to important sensor features, for instance high sensitivity, high birefringence, and fewer confinement losses.

\section{CONCLUSION}

A nonlinear Photonic Crystal Fiber (PCF) sensor is illustrated in this paper which shows high sensitivity and high birefringence along with reduced confinement losses for liquid analyte sensing applications. It has been shown in the numerical outcomes obtained that there can substantial improvements in relative sensitivity and birefringence when the sizes of air holes in the central region of the suggested PCF structures can be altered for different analytes. In addition, it was found in our study that there is a significant effect of $d_{1}$ and $d_{2}$ diameter dimensions on the overall performance of the suggested PCF with respect to birefringence and relative sensitivity. It has been found in our numerical findings that it is possible to attain a high birefringence of $10^{-3}$ and adequate sensitivity levels when the suggested nonlinear PCF sensor is at a wavelength of $1 \mu \mathrm{m}$. Consequently, the PCF structures that have been suggested have a greater ability to carry out liquid analyte sensing. This makes these PCF structures optimal for being used in biological and chemical applications $[8,31]$.

\section{REFERENCES}

[1] K. Saitoh, M. Koshiba, T. Hasegawa, E. Sasaoka, Chromatic dispersion control in photonic crystal fibres: application to ultra-flattened dispersion, Opt. Express 11(2003) 843-852.

[2] C. M. B. Cordeiro, M. A. R. Franco, G. Chesini, E. C. S. Barretto, R. Lwin, C. H. B. Cruz, M. C. J. Large, Microstructured-core optical fibre for evanescent sensing applications, Opt. Express 14(2006) 13056-13066.

[3] Y. Zhu, H. Du, R. Bise, Design of solid-core microstructured optical fiber with steering-wheel air cladding for optimal evanescent-field sensing, Opt. Express 14 (2006) 3541-3546.

[4] J. Limpert, T. Schreiber, S. Nolte, H. Zellmer, T. Tunnermann, R. Iliew, F. Lederer, J. Broeng, G. Vienne, A. Petersson, C. Jakobsen, High-power air-clad large-mode-area photonic crystal fiber laser, Opt. Express 11(2003) 818-823.

[5] T. Yang, E. Wang, H. Jiang, Z. Hu, K. Xie, High birefringence photonic crystal fiber with high nonlinearity and low confinement loss, Opt. Express 23 (2015) 8329- 8337.

[6] M. Vieweg, T. Gissible, S. Pricking, B.T. Kuhlmey, D.C. Wu, B.J. Eggleton, H. Giessen, Ultrafast nonlinear optofluidics in selectively liquid-filled photonic crystal fibers, Opt. Express 18 (2010) 25232-25240.

[7] E. K. Akowuah, H. Ademgil, S. Haxha, F. AbdelMalek, An Endlessly Single-Mode Photonic Crystal Fiber with Low Chromatic Dispersion, and Bend and Rotational Insensitivity, J. Lightwave Technol. 27 (2009) 3940-3947.

[8] A.M. Cubillas, S. Unterkofler, T.G. Euser, B.J.M. Etzold, A. C. Jones, P. J. Sadler, P. Wasserscheid, P. St. J. Russell, Photonic crystal fibres for chemical sensing and photochemistry, Chemical Society Reviews, 42 (2013) 8629-8648. 
[9] Y. L. Hoo, W. Jin, H. L. Ho, D. N. Wang, R. S. Windeler, Evanescent-wave gas sensing using microstructure fiber, Opt. Engineeering 41 (2002) 8-9.

[10] S. Liu, Y. Wang, M. Hou, J. Guo, Z. Li, P. Lu, Anti-resonant reflecting guidance in alcoholfilled hollow core photonic crystal fiber for sensing applications, Opt. Express, 21 (2013) 3169031697.

[11] Y. Yue, G. Kai, Zhi Wang, T. Sun, L. Jin, Y. Lu, C. Zhang, J. Liu, Y. Li, Y. Liu, S. Yuan, X. Dong, Highly birefringent elliptic-hole photonic crystal fibre with squeezed hexagonal lattice, Opt. Lett. 32 (2007) 469-471.

[12] G. P. Agrawal, Nonlinear Fiber Opt., fourth ed., Academic Press, San Diego, CA, 2006.

[13] T. M. Monro, W. Belardi, K. Furusawa, J. C. Baggett, N. G. R. Broderick, D. J. Richardson, Sensing with microstructured optical fibres, Meas. Sci. Technol. 12(2001) 854-858.

[14] H. W. Lee, M. A. Schmidt, P. Uebel, H. Tyagi, N. Y. Joly, M. Scharrer, P. St.J. Russell, Optofluidic refractive-index sensor in step-index fiber with parallel hollow micro-channel, Opt. Express 19 (2011) 8200-8207.

[15] J. Park, D. E. Kang, B. Paulson, T. Nazari, K. Oh, Liquid core photonic crystal fiber with low-refractive-index liquids for optofluidic applications, Opt. Express 22(2014) 17320-17330.

[16] H. Ademgil, Highly sensitive octagonal photonic crystal fiber based sensor, Optik International Journal for Light and Electron Opt. 125(2014) 6274-6278.

[17] Y. Yu, X. Li, X. Hong, Y. Deng, K. Song, Y. Geng, H. Wei, W. Tong, Some features of the photonic crystal fiber temperature sensor with liquid ethanol filling, Opt. Express 18(2010) 1538315388.

[18] M. Sharma, N. Borogohain, S. Konar, Index Guiding Photonic Crystal Fibers With Large Birefringence and Walk-Off, J. Lightwave Technol. 31(2013) 3339-3344.

[19] S.E.Kim, B.H.Kim, C.G. Lee, S. Lee, K. Oh, C.S. Kee, High birefringence photonic crystal fiber with high nonlinearity and low confinement loss, Opt. Express 20 (2012) 1385-1391.

[20] Y. Zhang, D. Li, Analysis of Birefringent Characteristics of Photonic Crystal Fibers Filled Magnetic Fluid, Advances in Brain Inspired Cognitive Systems Lecture Notes in Computer Science 7366(2012) 250-258.

[21] M. Pang, L. M. Xiao, W. Jin, Arismar Cerqueira S., Jr., Birefringence of Hybrid PCF and Its Sensitivity to Strain and Temperature, J. Lightwave Technology 30 (2012) 1422-1432.

[22] P. Ma, N. Song, J. Jin, J. Song, X. Xu, Birefringence sensitivity to temperature of polarization maintaining photonic crystal fibers, Optics \& Laser Technology 44 (2012) 1829-1833.

[23] A. Bjarkev, J. Broeng, and A. S. Bjarkev, Photonic Crystal Fibres, Kulver Academic, 2003.

[24] S. Yiou, P. Delaye, A. Rouvie, J. Chinaud, R. Frey, G. Roosen, P. Viale, S. Février, P. Roy, J. L. Auguste, J. M. Blondy, Stimulated Raman scattering in an ethanol core microstructured optical fiber, Opt. Express 13 (2005) 4786-4791.

[25] K. Saitoh, M. Koshiba, Numerical modeling of photonic crystal fibers, J. Lightwave Technol. 23 (2005) 3580-3590.

[26] C. M. Cordeiro, E. M. Dos Santos, C. H. Brito Cruz, C. J. de Matos, D. S. Ferreiira, Lateral access to the holes of photonic crystal fibers - selective filling and sensing applications, Opt. Express 14 (2006) 8403-8412.

[27] R. C. Kamikawachi, I. Abe, A. S. Paterno, H. J. Kalinowski, M. Muller, J. L. Pinto, J. L. Fabris, Determination of thermo-optic coefficient in liquids with fiber Bragg grating refractometer, Opt. Commun. 281(2008) 621-625. 
[28] Y. Huang, Y. Xu, A. Yariv, Fabrication of functional microstructured optical fibers through a selective-filling technique, Applied Phys. Lett. 85(2004) 5182-5184.

[29] M. Luo, Y. Liu, Z. Wang, T. Han, Z. Wu, J. Guo, W. Huang, Twin-resonance-coupling and high sensitivity sensing characteristics of a selectively fluid-filled microstructured optical fiber, Opt. Express 21(2013) 30911-30917.

[30] R. M. Gerosa, D. H. Spadoti, C. J. S. de Matos, L. S. Menezes, M. A. R. Franco, Efficient and short-range light coupling to index-matched liquid-filled hole in a solid-core photonic crystal fiber, Opt. Express 19 (2011) 24687-24698.

[31] S. Zheng, B. Shan, M. Ghandehari, J. Ou, Sensitivity characterization of cladding modes in long-period gratings photonic crystal fiber for structural health monitoring, Measurement 72 (2015) 43-51. 